\title{
Ferromagnetic resonance in submicron permalloy stripes
}

\author{
E. V. Skorohodov*1,2, R. V. Gorev ${ }^{1}$, R. R. Yakubov², E. S. Demidov², Yu. V. Khivintsev², \\ Yu. A. Filimonov ${ }^{3}$, and V. L. Mironov ${ }^{1,2 *}$ \\ ${ }^{1}$ Institute for Physics of Microstructures RAS, 603950, Nyzhny Novgorod, Russia \\ ${ }^{2}$ Lobachevsky State University, 603950, Nyzhny Novgorod, Russia \\ ${ }^{3}$ Kotel'nikov Institute of Radio-engineering and Electronics RAS, Saratov branch, 410019, Saratov, Russia \\ *Corresponding author: evgeny@ipmras.ru
}

\begin{abstract}
We present systematic experimental investigation and micromagnetic simulation of ferromagnetic resonance (FMR) in planar rectangular permalloy microstripes. The experimental microwave absorption was studied for different sample orientations in an external magnetic field. To analyze the FMR modes we developed the algorithm for the simulation of spectrum and spatial distribution of magnetization oscillations in dependence on swept external magnetic field based on numerical solution of Landau-Lifshitz-Gilbert equation. It was shown good agreement between experimental and model FMR spectra that enables the reliable visualization for spatial distributions of oscillating magnetization in modes of spin-wave resonances for different excitation conditions.
\end{abstract}

\section{Introduction}

The microwave properties of the ferromagnetic planar patterned structures (PPS) is the subject of the recent intensive investigations motivated by perspective applications in magnetoelectronics, spintronics and data processing [1-3]. In particular, the PPS consisting of ferromagnetic elements with different shape and spatial arrangement are considered as the effective filters for the ultra highfrequency electromagnetic radiation [4,5]. The spin wave resonances in PPS strongly depend on the internal fields connected with the shape anisotropy, exchange and magnetostatic interaction. As a result the absorption spectra are defined by the different geometric factors such as shape, size, aspect ratio and spatial arrangement of elements in PPS. This opens the wide opportunities for tuning of microwave absorption by changing the architecture of PPS using the nanolithography methods. In this regard, the special researches are focused on the study of FMR in rectangular microstripes, considered as one of the main structural elements of planar high-frequency microsystems. Due to the high shape anisotropy the microstripes have a uniform magnetic state and can be used in the microwave devices without external magnetizing. In particular, the PPS with strong magnetostatic interaction enables the realization of tuned filters with different absorption spectra, which can be switched by the external magnetic field [6].

Partially the investigations of the magnetization oscillations spectra depending on the size and shape of microstripes were reported in [6-13]. The special attention was paid to the analysis of magnetostatic standing waves and localized edge modes arising from the non-homogeneity of internal magnetostatic field near the boundaries of microstrip [10-13]. However, the systematic studies of exchange wave's contribution in FMR spectra for different microstrip orientations in an external magnetic field have not been performed.

On the other part, the dynamic properties of PPS systems are widely studied in different groups by micromagnetic modeling based on the numerical solution of the Landau-Lifshitz equation. In general, they simulate the spectra of eigenfrequencies based on the Fourier transformation of the relaxation oscillations of magnetization [13] and analyze only long-wavelength magnetostatic excitations. However, often the FMR is studied experimentally by the analysis of the microwave absorption at fixed frequency in dependence on the magnitude and direction of sweeping external 
dc magnetic field. Appropriate modeling of the spectra and spatial distributions of steady-state magnetization oscillations in external magnetic field for such experimental configuration were not discussed.

In current paper we present the results of comparative experimental measurements and micromagnetic simulations of magnetization oscillations in planar rectangular microstripes for different sample orientations in an external magnetic field. The main attention is given to the analysis of spatial distribution of magnetization oscillations in various modes of spin-wave resonances.

\section{Experiments and methods}

The array of permalloy $\left(\mathrm{Ni}_{80} \mathrm{Fe}_{20}\right)$ rectangular stripes $3000 \times 500 \times 30 \mathrm{~nm}^{3}$ (the separation between stripe's centers is about $6 \mu \mathrm{m}$ ) were fabricated by electron-beam lithography and lift-off process. At the first stage the $150 \mathrm{~nm}$ resist was deposited on Si substrate by centrifuging. Then initial mask in the form of rectangular stripes array was formed in negative electron resist using scanning electron microscope SUPRA 50VP with lithographic facility ELPHY PLUS. After that the irradiated areas of resist were removed in a selective organic solvent. At the next step the sample was covered by permalloy layer (30 $\mathrm{nm}$ thick) using magnetron sputtering. At the final stage the areas of permalloy layer except the strip array were removed in the lift-off process. The typical SEM image of strip array is presented in fig. 1(a).

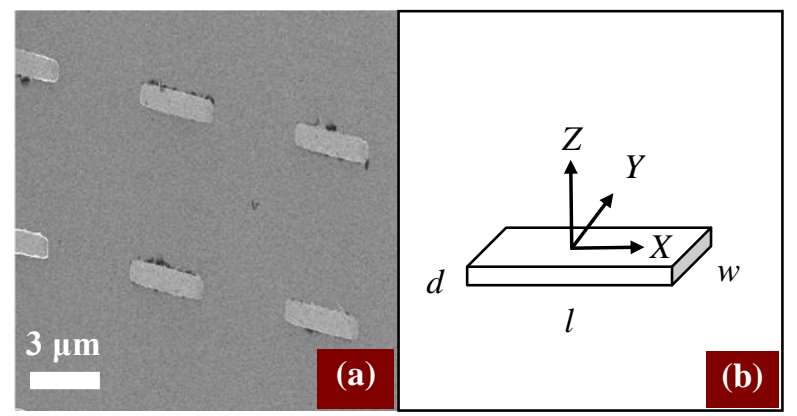

Fig. 1. (a) The SEM image of permalloy rectangular stripes. (b) The associated coordinate system, connected with the sample.

We have chosen the distance between the microstripes large enough to avoid the magnetostatic interaction between neighboring elements in the array, so the sample FMR spectrum corresponds to the spectrum of single microstrip. Note that all microstrip geometrical sizes are less than the path length of spin waves in permalloy that allows us to observe both magnetostatic and wechenge wave resonances (the formation of standing spin waves).

The FMR measurements were performed with Bruker EMX Plus-10/12 electron paramagnetic resonance spectrometer using a $\mathrm{TE}_{011}$ mode of cylindrical resonant cavity and dc magnetic field $(H)$ up to $2 \mathrm{~T}$. The polarized microwave magnetic field $h$ with frequency $9.8 \mathrm{GHz}$ was perpendicular to the $H$. The samples were driven through the resonance by sweeping the $H$.

Theoretically, the microwave absorption in microstrip was studied by computer micromagnetic modeling based on numerical solution of Landau-Lifshitz-Gilbert equation using the partly modified "Object Oriented MicroMagnetic Framework" (OOMMF) code [14]. We investigated the dependencies of microwave power absorption on the magnitude of swept $H$. Algorithm of numerical simulation includes follow stages. 1) The external magnetic field $H$ is applied. 2) The standing spin waves are excited by microwave magnetic field $h .3$ ) The dependence of averaged variable magnetization amplitude on the magnitude of external dc magnetic field is graphed. This dependence is a microwave power absorption spectrum since absorbed power is proportional to the sample averaged variable magnetization amplitude. In addition, to visualize the mode composition of resonant oscillations we calculated the time dependencies of spatial distributions for different magnetization components. The calculations were performed for the typical permalloy parameters: 
the saturation magnetization was $M_{\mathrm{S}}=8 \times 10^{5} \mathrm{~A} / \mathrm{m}$, the exchange stiffness was $\mathrm{A}=8.4 \times 10^{-12} \mathrm{~J} / \mathrm{m}$, and the damping constant was 0.01 (see [14]). We omitted magnetocrystalline anisotropy, assuming a polycrystalline structure of our samples, the perpendicular uniaxial anisotropy $K=3,9 \times 10^{4} \mathrm{~J} / \mathrm{m}^{3}$. The microwave frequency was $9.8 \mathrm{GHz}$. In calculations the microstripe $3000 \times 500 \times 30 \mathrm{~nm}^{3}$ was discretized into rectangular parallelepipeds with a square base of size $\delta=10 \mathrm{~nm}$ in the $x, y$ plane and height $h=30 \mathrm{~nm}$. The choice of geometrical dimensions and aspect ratio (the ratio of thickness, width and length) of microstripes was motivated by convenience for the experimental and theoretical study of the eigenmodes of magnetization oscillations. In case of high aspect ratio the magnetostatic interaction would give rise to noticeable shape anisotropy and substantial inhomogeneity of internal magnetic field that allows studying the influence of these effects on FMR spectrum.

\section{Results and discussion}

We investigated the FMR response in dependence on sample orientation relatively $\vec{H}$. In all experiments the exciting magnetic field $\vec{h}$ was directed in the sample plane since the oscillation excitation efficiency in this case is maximal. At the first stage we investigated the FMR for the case when the external magnetizing field was applied along the long side of microstripe ( $\mathrm{X}$ axis in fig. 1b). Fig. 2a shows experimental (dashed red curve) and numerically calculated (solid blue curve) FMR spectra for this configuration. It is seen that there is a good agreement between the experiment and simulation. As is seen from fig. $2 b$ the resonant oscillations corresponding to resonance fields 1 - 3 have complex spatial distributions, which are superposition of magnetostatic (long-wave) and exchange (short-wave) standing waves with different longitudinal wave numbers $k_{x}$. To explain the observed mode composition we calculated the spectra of spin waves for the microstripe in an external magnetic field directed along the $\mathrm{X}$ axis. The theory of the dipoleexchange wave's spectra was developed in [16-18]. Following the proposed approach we assumed that the surface magnetic moments are not fixed and the lower modes do not depend on the $z$ coordinate. It is known that the demagnetizing field of a rectangular parallelepiped is substantially nonuniform that leads to the complicated dependence of the variable component magnetization on coordinates $x$ and $y$. However, approximately the distribution of transverse component of the variable magnetization can be written as follows [18]:

$$
m_{y, z}(x, y) \approx M_{s} \cos \left(k_{x n} x\right) \cos \left(k_{y m} y\right),
$$

where $M_{s}$ is magnetization in saturation; $k_{x n}=\pi(n+1) / l$ and $k_{y m}=\pi(m+1) / w ; l$ and $w$ are the length and width of the microstripe respectively. The deviation of the variable component of the magnetization from the cosine function is $d / l$ (where $d$ is the microstripe thickness). On the other hand, the amendment to the spectrum is $(d / l)^{2}$, which is not substantial for the stripes with high aspect ratio. Thus, the spectrum of magnetization oscillations can be represented as follows [17,18]:

$$
\omega_{n m}^{2}=\gamma M_{s}\left(\omega_{H}^{n m}+J \omega_{M} k_{n m}^{2}\right)\left(\omega_{H}^{n m}+J \omega_{M} k_{n m}^{2}+\omega_{M} F_{m n}\right),
$$

where $\gamma$ is gyromagnetic ratio; $\omega_{M}=4 \pi M_{s} ; \omega_{H}^{n m}=\gamma H-\omega_{M} N_{n m} ; N_{n m}$ are demagnetizing factors for different modes in the microstripe; $F_{m n}$ are the matrix element of the dipole-dipole interaction; $J$ is the exchange stiffness constant. Using (2) and taking into account the frequency of the microwave oscillations $v_{0}=9.8 \mathrm{GHz}$, one can find the resonant magnetic fields for each FMR mode. As can be seen from fig. $2 b$, in the resonances we observe a superposition of two modes with low and high spatial frequencies (wave numbers). It is connected with the excitation of both dipole spin waves with small $k_{x n}$ as well as exchange waves with large $k_{x n}$. The dispersion relation in this case is nonmonotonic and there is the degeneracy in the spectrum. 


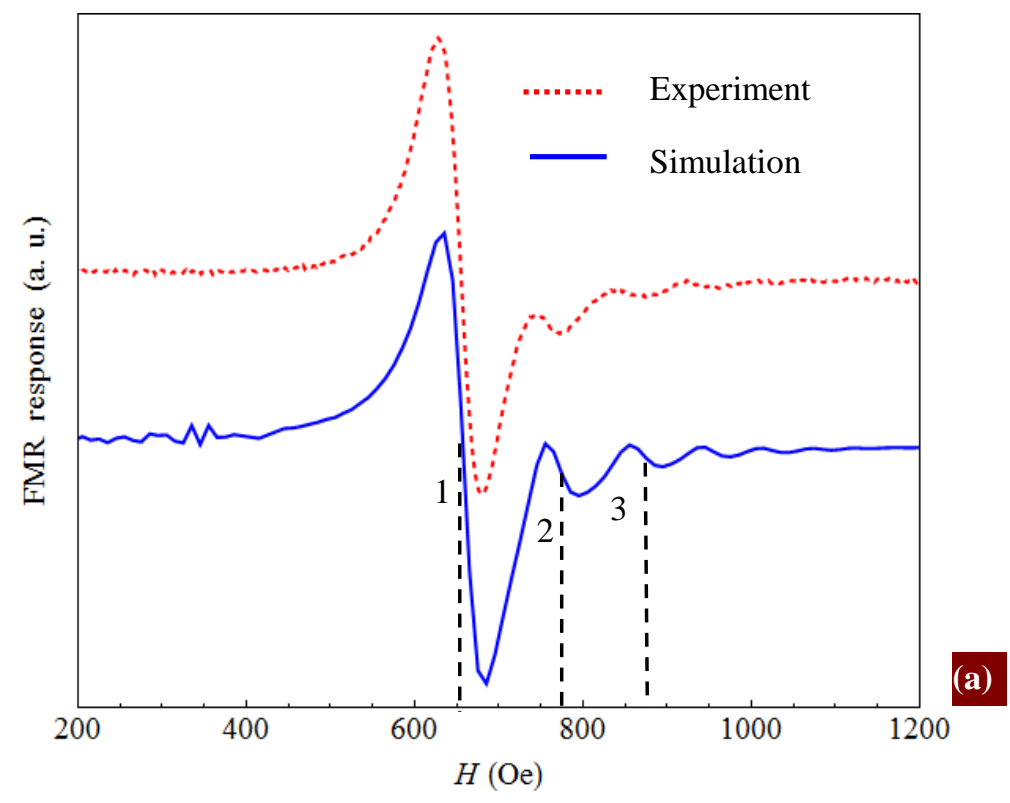

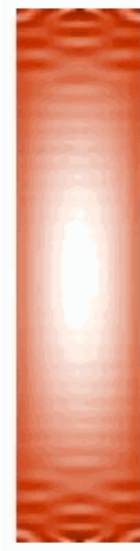

(1)

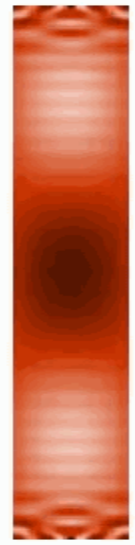

(2)

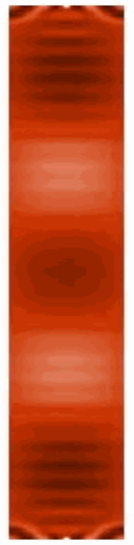

(3)

(b)

Fig. 2. (a) The experimental (dashed red curve) and simulated (solid blue curve) microstripe FMR spectra for the case when external magnetizing field applied along $\mathrm{X}$ axis and microwave field along Y axis. (b) The spatial distributions of the magnetization oscillations (amplitude of the zcomponent) in different resonant fields. (1) is for $H=660 \mathrm{Oe}$; (2) is for $H=770 \mathrm{Oe}$; (3) is for $H=870$ Oe.

The calculations show that the resonances are realized at $H=645$ Oe for the modes with $n=0$ and $n=84$ (resonance No 1); at $H=740$ Oe for the modes with $n=2$ and $n=82$ (resonance No 2); at $H=850$ Oe for the modes with $n=4$ and $n=78$ (resonance No 3) respectively. For these resonances $m$ spin wave mode number is zero $(m=0)$. The values of the resonance fields and numbers of longitudinal spin-wave modes are in good agreement with the results of micromagnetic simulations (fig. 2a).

The results for the other sample orientation (magnetizing field is applied along $\mathrm{Y}$ axis and microwave field along $X$ axis) are presented in fig. 3. In general, we observe a good agreement between the experiment and the simulation (see fig 3a), but there is one additional edge mode in model spectrum at resonant field $H=2140$ Oe. The analytical calculation shows that there is the degeneracy of resonance magnetic field with respect to mode number for modes with wave numbers $(n=2 ; m=0)$ and $(n=2 ; m=12)$ for resonance No $1 ;(n=0 ; m=0)$ and $(n=0 ; m=10)$ for resonance No $2 ;(n=4 ; m=4)$ and $(n=4 ; m=8)$ for resonance No 3 (see fig. 3b). For other modes 
the degeneracy is not observed. The resonances No 4 and No 5 correspond to the edge modes. The resonance No 4 has three half-waves, while the resonance No 5 has one half-wave spatial structure (fig. 3b).

Obviously, that the edge roughness has a big influence on edge mode structure for configuration when external magnetizing field is applied along short sample side (Y axis). To adjust the model spectrum for edge mode with resonant field $H=2140$ Oe we take into account edge roughness with $15 \mathrm{~nm}$ standard deviation (close to the observed experimentally). In this case edge modes are suppressed, while the structure of non-edge modes is not changed practically (fig. 4a and 4b).

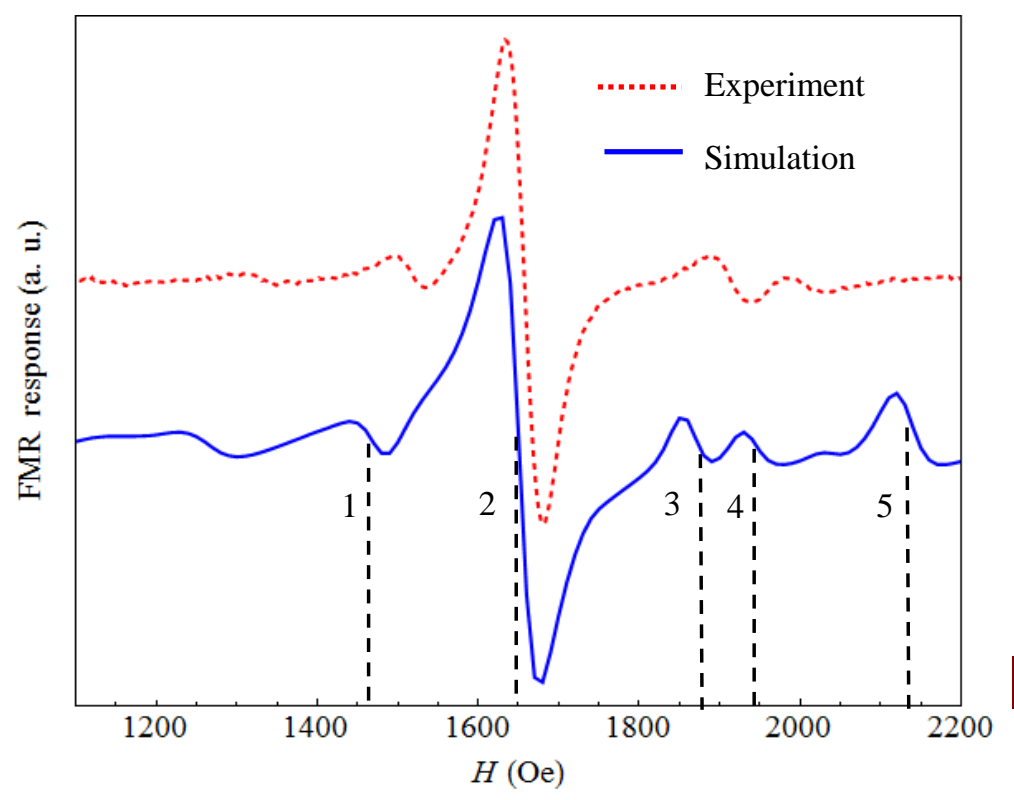

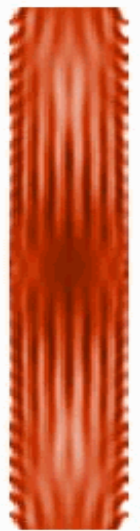

(1)

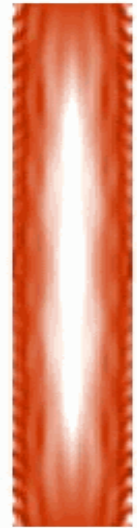

(2)

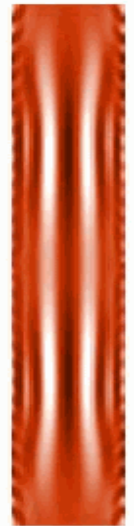

(3)

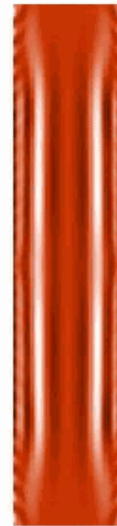

(4)

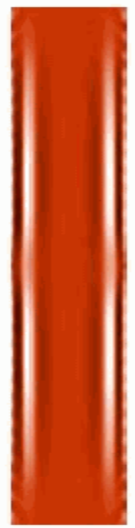

(b)

Fig. 3. (a) The experimental (dashed red curve) and simulated (solid blue curve) microstripe FMR spectra for the case when external magnetizing field applied along $\mathrm{Y}$ axis and microwave field along $\mathrm{X}$ axis. (b) The spatial distributions of the magnetization oscillations (amplitude of the $\mathrm{z}$ component) in different resonant fields. (1) is for $H=1493 \mathrm{Oe}$; (2) is for $H=1650 \mathrm{Oe}$; (3) is for $H=1870 \mathrm{Oe}$; (4) is for $H=1940 \mathrm{Oe}$; (5) is for $H=2140$ Oe. 


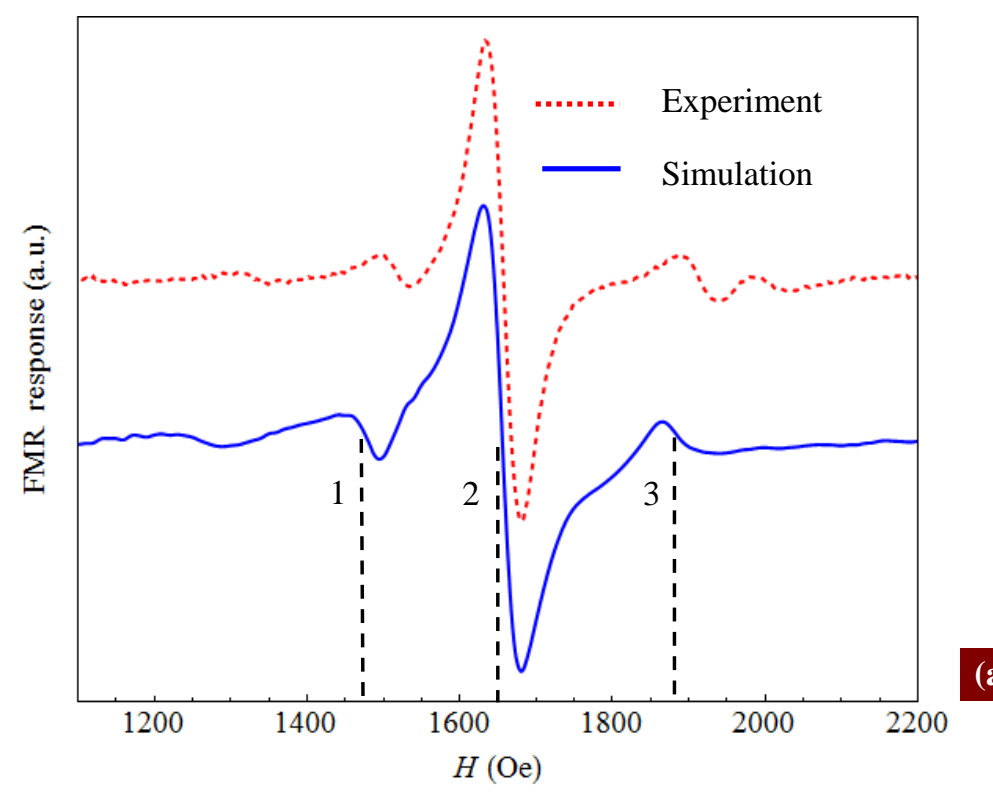

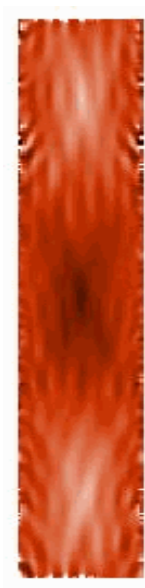

(1)

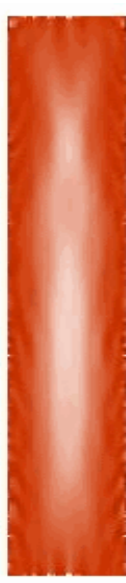

(2)

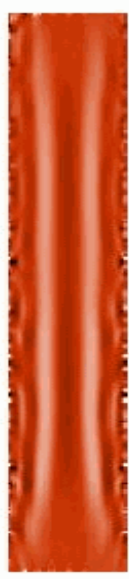

(3) (b)

Fig. 4. (a) The experimental (dashed red curve) and simulated (solid blue curve) microstripe FMR spectra for the case when magnetizing field applied along $\mathrm{Y}$ axis and microwave field along $\mathrm{X}$ axis. The microstripe edge rms roughness is $15 \mathrm{~nm}$. (b) The spatial distributions of the magnetization oscillations (amplitude of the $\mathrm{z}$-component) in different resonant fields. (1) is for $H=1493$ Oe; (2) is for $H=1650 \mathrm{Oe}$; (3) is for $H=1870 \mathrm{Oe}$

Fig. 5a shows experimental and simulated microstripe FMR spectra for the sample orientation when external magnetizing field is applied perpendicular to the sample plane ( $\mathrm{Z}$ axis in fig $1(\mathrm{~b})$ ). In this case we observed main resonance and series of spin-wave resonances locating left from main mode. The mode visualization shows that for resonances No 1-3 the amplitude of magnetization oscillations has half-wave spatial structure in Y direction and one-, two-, three half-wave structure in $\mathrm{X}$ direction respectively. The resonances No 4 and No 5 are the superposition of two different modes corresponding to the different directions of wave propagation, but in this case the degeneracy of resonance magnetic field from mode number is absent. For $H=10850$ Oe resonance modes are $(n=0 ; m=2)$ and $(n=18 ; m=0)$. For $H=10320$ Oe resonance modes are $(n=0 ; m=4)$ and $(n=34 ; m=0)$. 


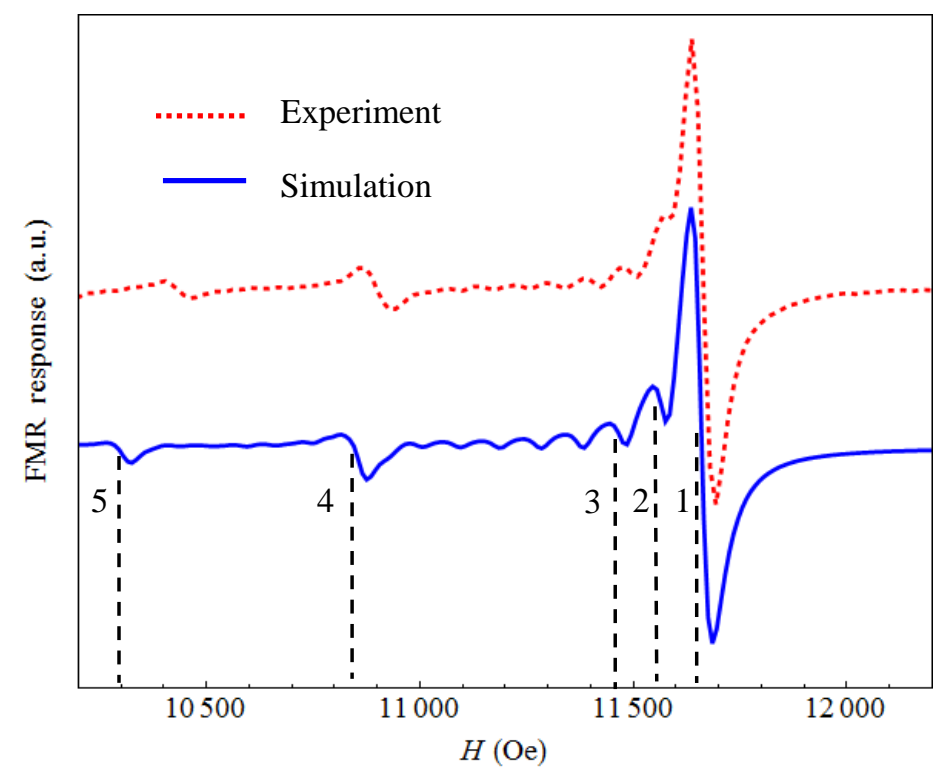

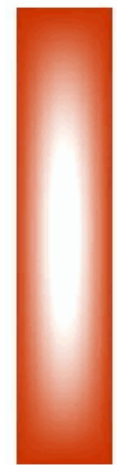

(1)

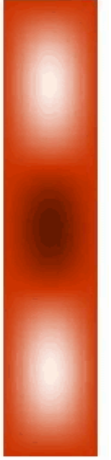

(2)

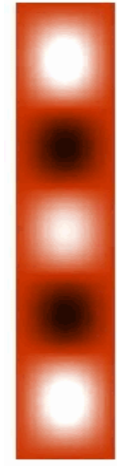

(3)

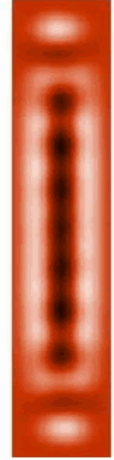

(4)

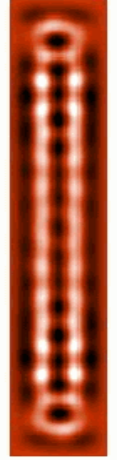

(5)

Fig. 5. (a) The experimental (dashed red curve) and simulated (solid blue curve) microstripe FMR for the case when magnetizing field applied along $\mathrm{Z}$ axis. (b) The spatial distributions of the magnetization oscillations (amplitude of the $\mathrm{z}$-component) in different resonant fields. (1) is for $H=11660 \mathrm{Oe}$; (2) is for $H=11580 \mathrm{Oe}$; (3) is for $H=11480 \mathrm{Oe}$; (4) is for $H=10850 \mathrm{Oe}$; (5) is for $H=10320$ Oe.

\section{Conclusion}

Thus we have developed the algorithm of micromagnetic simulations of forced magnetization oscillations, which enables the calculation of FMR spectra and the visualization of spatial structure for different sample resonant modes. In particular, we have tested this algorithm in comparative experimental and modeling investigations of FMR in permalloy rectangular $3000 \times 500 \times 30 \mathrm{~nm}^{3}$ microstripes for three different sample orientations in external magnetizing field. The good agreement between the experimental and model spectra gives us reason to believe that the model time dependences of magnetization oscillations correspond to the actual magnetic dynamics of the sample. We have performed model calculations of spatial structures of various resonance modes, together with analytical calculations of standing spin waves in our sample. It was shown that resonant oscillations have the complicated spatial structure corresponding to the superposition of long-wave and short-wave spin modes. For longitudinal magnetizing the main resonances correspond to the superposition of degenerated magnetostatic and exchange spin waves. On the 
other side, the localized edge modes play the noticeable role for the transverse sample magnetizing. These resonances are very sensitive to the sample edge state (defects of shape, roughness and etc.). In this case the fit of experimental and model FMR spectra can be used for the characterization of edge quality in arrays of magnetic microelements.

As the future development the designed model of micromagnetic simulation can be applied for the studies of influence of shape, magnetostatic and exchange interaction effects on FMR in arrays of various ferromagnetic nanostructures [19].

\section{Acknowledgements}

The authors are very thankful to A.A. Fraerman for the useful discussions and S.N. Vdovichev for the help in sample fabrication. We also thank M. J. Donahue and R. D. McMicheael (NIST, USA) for helpful councils. This work was supported by the Russian Foundation for Basic Research (project No 15-02-04462).

\section{References}

1. H. Zhang, A. Hoffmann, R. Divan, and P. Wang, Appl. Phys. Lett., 95, 232503 (2009).

2. V. Flovik, F. Macià, J. M. Hernàndez, R. Bručas, M. Hanson E. Wahlström. Phys. Rev. B, 92, 104406 (2015).

3. R. Adam, Yu. Khivintsev, R. Hertel, C. M. Schneider, A. Hutchison, R. Camley, and Z. Celinski, J. Appl. Phys., 101, 09F516 (2007).

4. B. K. Kuanr, R. Lopusnil, L. M. Malkinski, M. Wenger, M. Yu, D. Scherer II, R. Camley, and Z. Celinski, J. Appl. Phys., 103, 07C508 (2008).

5. L. Malkinski, M. Yu, A.Y. Vovk, D. Scherer II, L. Spinu, W. Zhou, S. Whittenburg, Z. Davis, and J.-S. Jung, J. Appl. Phys., 101, 09J110 (2007).

6. M. P. Wismayer, B. W. Southern, X. L. Fan, Y. S. Gui, R. E. Camley and C.-M. Hu, Phys. Rev. B, 85, 064411 (2012).

7. J. Jorzick, S. O. Demokritov, M. Bailleul, C. Fermon, B. Hillebrands, K. Guslienko, A. Slavin, D. Berkov, and N. Gorn, Phys. Rev. Lett., 88, 047204 (2002).

8. P. H. Bryant, Phys. Rev. B, 47, 11255 (1993).

9. N. Yu. Grigoryeva, D. A. Popov, and B. A. Kalinikos, Physics of Solid State, 56(9), 1806 (2014).

10. M. Bailleul, D. Olligs, and C. Fermon, Phys. Rev. Lett., 91, 137204 (2003).

11. R. D. McMichael and B. B. Maranville, Phys. Rev. B, 74, 024424 (2006).

12. C. Wen-Bing, H. Man-Gui, Z. Hao, O. Yu, and D. Long-Jiang, Chin. Phys. B, 19(8), 087502 (2010).

13. M. Buess, T. Haug, M. Scheinfein, and C.Bac, Phys. Rev. Lett., 94, 127205 (2005).

14. M. J. Donahue and D. G. Porter, Interagency Report No. NISTIR 6376, National Institute of Standards and Technology, Gaithersburg, (http://math.nist.gov/oommf/).

15. Yu. Yin, F. Pan, M.Ahlberg, M. Ranjbar, A. Mojtaba, Ph. Durrenfeld, M.Haidar, Ya Zhai, J. Akkerman, A. Delin,L. Bergqvist, and R. Dumas, Phys. Rev. B, 92, 024427 (2015).

16. B. A. Kalinikos and A. N. Slavin, J. Phys. C, 19(35), 7013 (1986).

17. K. Yu. Guslienko and A. N. Slavin, J. Appl. Phys., 87, 6337 (2000).

18. K. Yu. Guslienko, R. W. Chantrell and A. N. Slavin, Phys. Rev. B, 68, 024422 (2003).

19. V.L. Mironov, E.V. Skorohodov, and J.A. Blackman, J. Appl. Phys., 115, 184301 (2014). 